\title{
LA PRESENCIA DE LA MUJER DEPORTISTA EN LA TELEVISIÓN
}

\author{
THE PRESENCE OF SPORTSWOMEN IN TELEVISION
}

\author{
Lilyan VEGA RAMÍREZ \\ Universidad de Alicante \\ M. ${ }^{\mathrm{a}}$ Alejandra Ávalos RAmos \\ Universidad de Alicante
}

Recibido: 11/01/2016

Aceptado: 31/03/2016

\section{Resumen}

La difusión del deporte en los medios de comunicación es algo fundamental para su evolución, el buen uso de los mismos podría contribuir a sociedades más equitativas en cuanto al género. El objetivo de este estudio es analizar la difusión de noticias deportivas en la televisión desde una perspectiva de género. La metodología utilizada es descriptiva y tiene un enfoque cuantitativo, realizándose una observación directa del contexto a estudiar: una televisión pública y dos televisiones privadas. Para la recogida de la información se ha empleado una plantilla de observación. Los resultados señalan que la divulgación de las noticias deportivas femeninas es significativamente menor con respecto a la difusión de las masculinas. Las consecuencias de esta situación de desigualdad podrían repercutir en la falta de promoción, de valoración y de captación del deporte femenino que a día de hoy se encuentra infravalorado por los medios de comunicación masiva.

Palabras clave: género, deporte, difusión, medios de comunicación, igualdad.

\begin{abstract}
The spread of sport in the mass media is fundamental to its evolution; good use of them could contribute to more equitable societies in gender. The aim of this study is to analyse the spread of sports news on television from a gender perspective. The methodology used is descriptive and has a quantitative approach, performing a direct
\end{abstract}


observation to study context: public television and two private televisions. For the collection of information has been employed a sheet for the observation. The results show that the disclosure of female sports news is significantly lower compared to the diffusion of male. The consequences of this unequal situation could affect the lack of promotion, appraisement and catchment of women's sport that today is undervalued by the mass media.

Keywords: gender, sport, diffusion, media, equality. 


\section{INTRODUCCIÓN}

La conducta de una sociedad puede verse conmovida por la información recibida a través de los medios de comunicación, en este sentido Fraga refiere que los medios de comunicación de masas en una sociedad moderna son un elemento esencial en la formación de opinión de las personas. Estos medios cimientan nuevas formas de pensar, ser, estar y hacer, aportando estándares económicos y valores sociales, además de fomentar el consumo y la participación en la vida política, entre otros aspectos a reflexionar (Domínguez; Toro).

La televisión actualmente es uno de los principales medios de comunicación masiva (Domínguez), todos los hogares poseen dos o más televisores dentro de las casas, así como los negocios, los bares, los restaurantes, los lugares de ocio, los aeropuertos entre otros, también cuentan al menos con un aparato de televisión (Cáceres, Ruiz, Brändle; Domínguez). A través de este medio de comunicación podemos estar informados de los sucesos que acontecen tanto a nivel nacional como internacional, asimismo pueden ser usados como una herramienta de ocio, entretenimiento y diversión (Domínguez; Ríos, Mata, Gómez).

Uno de los temas que tiene una alta demanda en los medios de comunicación masiva son los deportes, De Moragas refiere que el deporte es una fuente ilimitada de personajes y de evidencias que sirven a los medios de comunicación para reflejar el éxito, el fracaso, la suerte y la desgracia, entre otros aspectos. Hoy en día las cadenas de televisión dentro de sus informativos dedican un bloque para tratar temas deportivos, con presentadores y periodistas especializados. Los contenidos deportivos emitidos por estos medios están relacionados mayoritariamente con el fútbol masculino, dejando poco tiempo para las demás disciplinas y menos aún para las relacionadas con las disciplinas femeninas.

La imagen que se proyecta principalmente de la mujer en general y de la deportista en particular en los medios de comunicación masiva, y especialmente en la televisión, es de una mujer joven identificada como objeto sexual que no tiene nada que ver con su trayectoria vital o profesional (Fraga; López; Martínez). El aspecto físico predomina ante el deporte y el rendimiento 
viéndose favorecida aquella deportista que cumple con los cánones de belleza actuales, apareciendo mencionada más frecuentemente en los medios de comunicación y pudiendo conseguir patrocinios fácilmente (López).

Si nos remontamos a la historia del deporte desde su origen, este no era un mundo de mujeres, incluso los hombres no soportaban su presencia, por lo que la trayectoria deportiva se inicia con la lucha por los derechos de las mujeres para acceder al mundo deportivo (Gutiérrez). Esta situación puede estar relacionada con el tipo de estereotipo de una mujer no agresiva y débil, cuyo papel principal era la maternidad y la atención de la familia, rol que se mantuvo por siglos, perjudicando a las mujeres en la participación de la práctica deportiva, ya que esta práctica se consideraba competitiva y agresiva y una actividad propia para los hombres (Tortosa, Vega). García refiere este hecho a la sociedad griega donde se crearon los Juegos Olímpicos, y donde la mitología dominante atribuía a los dioses las cualidades de actividad, vigor y fuerza y a las diosas los rasgos de belleza, sexualidad y pasividad. Estos acontecimientos han relegado al deporte femenino a un segundo plano desde la antigüedad, donde solo podían participar en los Juegos griegos de Hera, que se celebraban después de los Juegos Olímpicos.

Conocido es que Pierre de Coubertin, al restablecer los Juegos Olímpicos modernos, reservó a las mujeres el «honor» de «coronar al vencedor» excluyéndolas de la participación competitiva. Así, en los juegos de 1900, la mujer fue admitida en algunas pruebas como golf, tenis, tiro con arco, vela y patinaje (López, Alvariñas). Los movimientos feministas promovieron a mediados del siglo XX, que las mujeres pudieran participar en otras modalidades, algunas de ellas propias del género masculino, produciéndose en muchos casos que las deportistas sufrieran discriminación social. Las mujeres eran criticadas de «marimachos» si participaban en disciplinas consideradas de «hombres». La imagen y el rol de la mujer deportista han ido cambiando a partir de los años 70 favoreciéndose en los últimos tiempos. Hoy la práctica deportiva femenina está mucho más generalizada tanto en el campo del alto rendimiento como en actividades recreativas de ocio o actividades físicas de salud, estando la presencia de la mujer en estos ámbitos más en consonancia con la evolución social (Merino). En esta línea, las olimpiadas son un reflejo de los avances de la incorporación de la mujer en el deporte, una muestra de ello es que en los últimos Juegos Olímpicos (Londres 2012) la participación femenina fue de un $45 \%$ del total de los deportistas, consiguiéndose que por primera vez todos los países inscribieran al menos una mujer en sus delegaciones (COI). A pesar de la incorporación que las deportistas españolas han tenido durante los últimos 25 años (Gutiérrez) y de los logros deportivos conseguidos a nivel 
colectivo (hockey, balonmano, baloncesto y natación sincronizada) e individual (natación, bádminton y tenis), todavía el deporte es posiblemente, uno de los medios donde el hombre es considerado superior (Bernstein). En este sentido, también se evidencia, como hemos mencionado anteriormente, la escasa presencia de la mujer en los medios de comunicación a nivel nacional e internacional. El informe de la Cuarta Conferencia Mundial sobre la Mujer (ONU 1996) propone potenciar la plena participación de las mismas en condiciones igualitarias en todas las esferas de la sociedad eliminando todas las formas de discriminación.

Por todo ello, nos hemos planteado que el objetivo de esta investigación sea analizar la difusión de las deportistas en los telediarios de mayor audiencia, desde una perspectiva de género.

\section{MÉTODO}

El estudio llevado a cabo es descriptivo y tiene un carácter cuantitativo, realizándose una observación directa de una situación concreta en un contexto específico. Para la recopilación de los datos se ha utilizado una plantilla de observación donde se atiende a los aspectos del número de noticias deportivas emitidas y al tiempo dedicado a las mismas, diferenciando las noticias y el tiempo según fueran sucesos de deporte femenino o masculino. La observación se llevó a cabo en tres cadenas de televisión, escogiendo dos cadenas privadas y una tercera cadena pública. La selección de los telediarios de las cadenas privadas se realizó bajo el criterio de los niveles de audiencia siendo los de la sesión nocturna los de mayor repercusión. El período de observación y registro se ha llevado a cabo durante 31 días, desde el 24 de octubre hasta el 23 de noviembre de 2015, cabe señalar que el 14 de noviembre las noticias se dedicaron exclusivamente a los atentados producidos en París por lo que se han descrito 30 días. El análisis de los datos se ha realizado con el programa informático Excel de Windows 2013.

\section{RESULTADOS}

En este apartado presentamos los resultados obtenidos en relación al número de noticias emitidas durante el periodo de observación de las tres cadenas y su diferenciación según el género (Tabla 1), así como el número de horas de emisión de las noticias deportivas y su distribución según género (Tabla 2).

En primer lugar, como se refleja en la Tabla 1, las tres cadenas de televisión emiten el mismo número de noticias deportivas independientemente del tipo de cadena que sea, privada o pública $(33,33 \%)$ y en segundo lugar, si 
tenemos en cuenta el número de noticias según el género, la representación de las deportistas (20\%) en los telediarios es muy inferior a la cifra obtenida en el deporte masculino (80\%).

En cuanto al tipo de cadena y según el género tratado en las noticias, todas las cadenas televisivas tienen una representación en número, mayoritaria del deporte masculino. En el caso de la televisión pública (TVE) cuenta con una difusión del contenido deportivo relacionado con los hombres del $56,66 \%$, siendo la emisión deportiva de las mujeres del 43,33\%. Si señalamos los resultados obtenidos de las cadenas privadas, observamos que en la cadena de Tele 5 los hombres ocupan un 86,66\% de noticias deportivas frente al escaso número de noticias de mujeres, un $13,33 \%$. Con respecto a la cadena de Antena 3, la tendencia de ocupación de noticias deportivas masculinas sigue creciendo teniendo en este caso una representación del 96,66\%. Por el contrario, las emisiones del deporte femenino son casi inexistentes con una representación mínima del 3,33\%.

Tabla 1. Número de noticias deportivas emitidas televisivamente según género, durante 30 días

\begin{tabular}{|l|c|c|c|c|c|c|}
\hline $\begin{array}{c}\text { Cadena } \\
\text { televisiva }\end{array}$ & $\begin{array}{c}\text { Noticias } \\
\text { deportivas }\end{array}$ & $\begin{array}{c}\text { Noticias } \\
\text { femeninas }\end{array}$ & $\begin{array}{c}\text { Noticias } \\
\text { masculinas }\end{array}$ & $\begin{array}{c}\text { \%Noticias } \\
\text { deportivas }\end{array}$ & $\begin{array}{c}\text { \%Noticias } \\
\text { femeninas }\end{array}$ & $\begin{array}{c}\text { \%Noticias } \\
\text { masculinas }\end{array}$ \\
\hline Antena 3 & 30 & 1 & 29 & $33,33 \%$ & $3,33 \%$ & $96,66 \%$ \\
\hline Tele 5 & 30 & 4 & 26 & $33,33 \%$ & $13,33 \%$ & $86,66 \%$ \\
\hline TVE & 30 & 13 & 17 & $33,33 \%$ & $43,33 \%$ & $56,66 \%$ \\
\hline TOTAL & 90 & 18 & 72 & & $20 \%$ & $80 \%$ \\
\hline
\end{tabular}

En relación con las horas de emisión de las noticias deportivas (Tabla 2), se refleja que la cadena pública TVE (3h 47') destina un tiempo de emisión a la sección de deportes superior al tiempo dedicado en las dos cadenas privadas, siendo Tele 5 la cadena que ocupa el segundo lugar en este sentido (2h 25') y Antena 3 ( $2 \mathrm{~h} 05^{\prime} 3 \mathrm{l}^{\prime}$ ) la cadena que invierte el menor tiempo en las noticias deportivas, en relación con todas ellas.

Si analizamos el tratamiento del deporte por género el resultado es muy significativo refiriéndonos, a la escasa y casi nula presencia de la mujer deportista en los medios con 11' y 13" (2,25\%) de dedicación frente a las 2h 05' y $31 "(97,74 \%)$ que las televisiones invierten en noticias masculinas.

Si desglosamos los resultados por cadenas, la televisión pública TVE destina $10^{\prime}(4,40 \%)$ de la sección deportiva al deporte femenino cifra que a diferencia de las 3 h y 37' (95,60\%) que destina al deporte masculino, es 
ínfimamente menor. Asimismo, y con un resultado todavía menor al anterior, la cadena de Tele 5 emite l' con 02 " $(0,72 \%)$ de deporte femenino frente a las 2 h 22 ' y 58 " (99,28\%) que dedican a los deportistas varones y sus disciplinas.

Por último, se presentan los datos de la cadena privada de Antena 3 cuya difusión de noticias de deportistas mujeres es casi nula durante los 30 días de análisis con apenas 11 " de emisión $(0,15 \%)$ frente a la presencia de noticias deportivas masculinas cuya duración es de 2h 05' y 20" (99,85\%).

Tabla 2. Tiempo total de horas de emisión televisiva de noticias deportivas según género, durante 30 días

\begin{tabular}{|c|c|c|c|c|c|c|}
\hline $\begin{array}{l}\text { Cadena } \\
\text { televisiva }\end{array}$ & Horas & Mujeres & Hombres & $\begin{array}{l}\text { \%emisión } \\
\text { segundos }\end{array}$ & $\begin{array}{c}\text { \% emisión } \\
\text { mujeres }\end{array}$ & $\begin{array}{c}\text { \%emisión } \\
\text { hombres }\end{array}$ \\
\hline Antena 3 & $2 \mathrm{~h} \mathrm{05’31"}$ & $11 "$ & $2 \mathrm{~h} 05^{\prime} 20^{\prime \prime}$ & $2528 \%$ & $0,15 \%$ & $99,85 \%$ \\
\hline Tele 5 & $2 \mathrm{~h} 24^{\prime}$ & 1'02" & 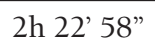 & $29 \%$ & $0,72 \%$ & $99,28 \%$ \\
\hline TVE & $3 \mathrm{~h} 47^{\prime}$ & $10^{\prime}$ & $3 \mathrm{~h} 37^{\prime} 00^{\prime \prime}$ & $45^{\prime} 71 \%$ & $4,40 \%$ & $95,60 \%$ \\
\hline TOTAL & 8h 16'31" & 11'13" & $8 \mathrm{~h} \mathrm{05'} 18^{\prime \prime}$ & & $2,25 \%$ & $97,74 \%$ \\
\hline
\end{tabular}

\section{DISCUSIÓN}

El objetivo de este estudio ha sido analizar la presencia de la mujer deportista en un medio de comunicación masivo como es la televisión. Destacando los resultados más relevantes podemos afirmar que en las tres cadenas de televisión analizadas la difusión del deporte femenino es muy inferior a las emisiones de noticias deportivas masculinas. Este hecho viene siendo estudiado a nivel internacional desde hace décadas, tal como se demuestra en un estudio realizado en EEUU por Messner y Cooky donde solo aparece un 6,3\% de representación femenina en la sección de deportes. Asimismo, en una investigación realizada en España a través del Proyecto de Monitoreo Global de Medios se evidencia que en las noticias deportivas televisivas los hombres están representados en un 99\% con respecto a las deportistas, al igual que en el estudio de Latorre y otros, datos que también reflejan la situación obtenida en nuestra investigación.

En cuanto al número de noticias emitidas sobre deporte femenino la cadena pública se acerca en número a las masculinas; cumpliendo así con el criterio de igualdad en las emisiones de acontecimientos deportivos; sin embargo nos encontramos con una realidad encubierta ya que el tiempo destinado a las diferentes noticias del espacio dedicado a la mujer deportista es muy inferior al destinado a los hombres, por lo que la dedicación al tiempo deportivo femenino sigue siendo minoritaria. Las cifras en cuanto a las cadenas 
privadas todavía son más alarmantes. Ninguna cadena se acerca ni en número de noticias ni en tiempo dedicado a las distintas disciplinas deportivas en su categoría de género. Existe una descompensación en cuanto a la cantidad de noticias y a su tiempo de emisión entre los hombres y las mujeres. Todos estos hallazgos coinciden con un estudio realizado por el Consejo Audiovisual de Andalucía donde se analizaron dos cadenas autonómicas, dos cadenas públicas y una cadena de televisión privada y donde se refleja que exclusivamente el 3,4\% de noticias deportivas fueron de disciplinas femeninas.

Esta situación discriminatoria no solo se da en la televisión, también existe un trato desigual hacia el deporte, en cuanto al género, en otros medios de difusión masiva como la prensa escrita. Este hecho se viene repitiendo a lo largo de la historia donde la prensa escrita no destina ni invierte en la difusión ni en el tratamiento igualitario del deporte femenino como se aprecia en un estudio longitudinal realizado en España, donde solo el 5,11\% de los periódicos dedican información a las deportistas (Sainz de Baranda). En un estudio reciente realizado en el País Vasco en 2013 (Aspic), donde se analizó la prensa generalista vasca y periódicos de información deportiva como son el Marca y el Mundo Deportivo quedó reflejado que las noticias deportivas femeninas publicadas solo representaban el 5,9\% del total, por lo que queda presente que la situación no ha evolucionado en absoluto.

Una de las causas que aducen los medios de comunicación en relación con la casi inexistente presencia de la mujer es la falta de éxitos deportivos alcanzados por las deportistas (López). En este sentido, esta justificación no se sostiene ya que la participación de las mujeres españolas en la última olimpiada de 2012 fue del 40,30\% y en cuanto al medallero, el 64,70\% fue obtenido por mujeres (COE). Otro de los motivos que podría relacionarse con la escasa difusión del deporte femenino en los medios es la poca participación de las niñas y las adolescentes en dichas actividades ya que sus prioridades suelen ser académicas, sociales y sentimentales entre otras (García, Llopis).

La ONU (1996), hace veinte años atrás, ya afirmó y difundió la necesidad de realizar un gran esfuerzo por parte de los medios de comunicación, para que esta situación se modificara y se pudiera romper con la falta de equidad en el tratamiento de la mujer, apoyándola y promocionándola en todos los ámbitos tanto en el social, en el económico, en el cultural como en el deportivo. A pesar de las oportunidades que pueden ofrecer los medios de comunicación para la evolución y el progreso de la mujer (De los Ríos, Martínez), en el caso del deporte femenino no están contribuyendo a este avance tal como se ha demostrado en nuestro estudio. 
Esta investigación no pretende generalizar los resultados a nivel nacional ya que solo se han analizado tres cadenas televisivas. Consideramos este trabajo como una primera aproximación para poder realizar futuros estudios sobre la temática tratada, abarcando un mayor número de cadenas televisivas e incluso poder analizar otros medios de comunicación masiva como puede ser la prensa escrita.

\section{CONCLUSIONES}

Las mujeres desde la antigüedad se han visto relegadas no solo en la participación deportiva sino en la presencia en los medios de comunicación, a pesar de la evolución actual del deporte femenino.

Los medios de comunicación ocupan un lugar fundamental para la difusión de los contenidos deportivos, la falta de tratamiento del deporte femenino en los mismos hace que este no se vea beneficiado en cuanto a su promoción, a su valoración y a su captación.

La cadena de televisión pública TVE difunde más noticias deportivas femeninas en número y tiempo que las cadenas privadas. A pesar de esto ninguna cumple con las políticas igualitarias lanzadas desde los organismos gubernamentales.

La presencia mayoritaria de noticias deportivas masculinas podría tener consecuencias negativas para la población femenina, ya que no tendrán modelos de referencia para la práctica deportiva y por otro lado se podrán dar mayores casos de abandono por falta de reconocimiento.

\section{REFERENCIAS BIBLIOGRÁFICAS}

Aspic. Asesores, prensa, imagen y comunicación. Presencia y tratamiento del deporte femenino en la prensa generalista vasca y en periódicos de información deportiva. Euskadi: Dirección de Juventud y Deportes del Gobierno Vasco, 2013.

Bernstein, Alina. «Is it time for a victory lap? Changes in the media coverage of women in sport». International Review for the Sociology of Sport 37.3-4 (2002): 415-428.

Cáceres Zapatero, María Dolores, José Antonio Ruiz San Ramón y Gaspar Brändle Señán. «El uso de la televisión en un contexto multipantallas: viejas prácticas en nuevos medios». Anàlisi 43 (2011): 21-44.

COE. Resultados Juegos Olimpicos Londres 2012. <http://www.coe.es/2012/Home Federaciones.nsf/FBuscadorResultadosFederacion?ReadForm\&tipoevent o=JJ.OO\&subtipo=Verano\&evento=Londres\%202012@@818\&buscar=1>, consultado el 10-12-2015. 
COI. Factsheet London 2012 Facts \& Figures. <http://www.olympic.org/Documents/Reference_documents_Factsheets/London_2012_Facts_and_Figureseng.pdf>, consultado el 10-12-2015.

Consejo Audiovisual de Andalucía. Estudio sobre género y deporte en televisión. Andalucía: Consejo Audiovisual de Andalucía, 2008.

De los Ríos, M. ${ }^{a}$ José y Joaquina Martínez Almería. «La mujer en los medios de comunicación». Comunicar 9 (1997): 97-104.

De Moragas Spà, Miguel. «Deporte y medios de comunicación sinergias crecientes». Telos: Cuadernos de Comunicación, Tecnología y Sociedad 38 (1994): 58-62.

Domínguez Goya, Emelia. Medios de comunicación masiva. México: Red tercer milenio, 2012.

Fraga Pérez, Cristina (2006). «Las mujeres y los medios de comunicación. Una relación controvertida». Comunicación e Ciudadanía 1 (2006): 1-9.< http://nova. observatoriodosmedios.org/imxd/noticias/doc/1236340428artigo37.pdf>, consultado el 19-06-2008.

García Ferrando, Manuel. Aspectos sociales del deporte. Una reflexión sociológica. Madrid: Consejo Superior de Deportes, 1990.

García Ferrando, Manuel y Ramón Llopis Goig. Ideal democrático y bienestar personal. Madrid: CIS, 2011.

Gutiérrez Pequeño, José Miguel. «Mujer, deporte y medios de comunicación». Mujer, Deporte y Medios de Comunicación 2 (2002): 259-264.

Latorre Román, Pedro Ángel, Miguel Ángel González Redondo, Alfonso Javier Moreno Padilla, María Dolores Sierra Jiménez, Francisco Javier Carmona del Jesús, Dolores Molina Gómez... Luisa Vergillos Carrillo. «Mujer, deporte y medios de comunicación». Revista Digital de Buenos Aires 11.106 (2007):1.

López Díez, Pilar. Deporte y mujeres en los medios de comunicación: sugerencias y recomendaciones. Madrid: Consejo Superior de Deportes, 2011.

López Villar, Cristina y Miriam Alvariñas Villaverde. «Anàlisis mostrals des d'una perspectiva de gènere en revistes d'investigació de ciències de l'activitat física i de l'esport espanyoles». Apunts: Educació Física i Esports 106 (2011): 62-70.

Martínez Verdú, Remedios. «Mujeres y medios de comunicación». Comunicación y Xénero. Eds. Lois Álvarez Pousa, Ana Belén Puñal Rama y Joám Evans Pim. Galicia: Colexio Profesional de Xornalistas de Galicia, Observatorio Galego dos Medios, 2007, 37-44.

Merino Mandly, Antonio. Mujer y Deporte: una fórmula de integración en la sociedad actual. Jaén: Diputación provincial Plan de Formación Deportiva, 2007.

Messner, Michael y Cheryl Cooky. Gender in televised sport. News and highlights shows, 1989-2009. California: Center for Feminist Research, University of Southern California, 2010. 
ONU. Informe sobre la cuarta conferencia mundial sobre la mujer. Nueva York: Naciones Unidas, 1996.

Proyecto de Monitoreo Global de Medios. ¿Quién figura en las noticias? Barcelona: Asociació de Dones Periodistes de Catalunya, 2010.

Ríos Ariza, José Manuel, Antonio Mata Terrón y Elba Rosa Gómez Barajas. «Investigación sobre el uso de la televisión por estudiantes de Educación de universidades hispanoamericanas». Profesorado. Revista de Curriculum y Formación del Profesorado 15.1 (2011): 305-323.

Sainz de Baranda Andújar, Clara. Mujeres y deporte en los medios de comunicación. Estudio de la prensa deportiva española (1979-2010) (Tesis Doctoral). Madrid: Universidad Carlos III, 2013.

Toro Castillo, Bárbara. «Medios masivos de comunicación: una construcción de la realidad». Revista Pequén 1.1 (2011): 108-119.

Tortosa Martínez, Juan y Lilyan Vega Ramirez. «Mujeres mayores y actividad física». Feminismo/s 21 (2013): 241-261. 